\title{
The use of microspectrofluorimetry for the characterization of lake pigments
}

\author{
Ana Claro ${ }^{\text {a,b }}$, Maria J. Melo ${ }^{a, b, *}$, Stephan Schäfer ${ }^{b}$, J. Sérgio Seixas de Melo ${ }^{c}$, \\ Fernando Pina $^{a}$, Klaas Jan van den Berg ${ }^{\mathrm{d}, * *}$, Aviva Burnstock ${ }^{\mathrm{e}}$ \\ ${ }^{\text {a }}$ REQUIMTE-CQFB and Faculty of Sciences and Technology of the New University Lisbon, Campus da Caparica, Portugal \\ ${ }^{\mathrm{b}}$ Department of Conservation and Restoration, New University Lisbon, Campus da Caparica, Portugal \\ ${ }^{\mathrm{c}}$ Department of Chemistry, University of Coimbra, Coimbra, Portugal \\ ${ }^{\mathrm{d}}$ ICN-Instituut Collectie Nederland, Netherlands Institute for Cultural Heritage, P.O. Box 76709, Amsterdam, Netherlands \\ ${ }^{\mathrm{e}}$ Department of Conservation and Technology, Courtauld Institute of Art, Somerset House, London WC2R ORN, United Kingdom \\ Received 8 December 2006; received in revised form 30 March 2007; accepted 30 July 2007 \\ Available online 11 August 2007
}

\begin{abstract}
In this paper, the potential of confocal microfluorescence spectroscopy is explored for the characterization of selected red lake pigments and paints based on alizarin, purpurin and eosin (weak, medium and strong emitters). The anthraquinone pigments have been used since ancient times by artists, and eosin lakes were used by impressionist painters. Reconstructions of artists paints based on 19th century recipes are examined. The paints were made using the lake pigments bound in a range of binding media including gum arabic, collagen, a vinyl emulsion and linseed oil. The acquisition of the spectra is rapid, with high spatial resolution and the data reliable and reproducible. Together with full emission spectra, it was possible to acquire well-resolved excitation spectra for purpurin, alizarin and eosin based colors. The present investigation suggests that micro-emission fluorescence can also be used as a semi-quantitative method for madder lake pigments, enabling the determination of purpurin lake ratio in a mixture of purpurin and alizarin, which is important for provenance studies. The data obtained with microfluorescence emission with those acquired with fiber-optic fluorimetry are compared. The spatial resolution used, $8 \mu \mathrm{m}$, is appropriate for the analysis of individual pigments particles or aggregates in a paint film. Micro-emission molecular fluorescence proved to be a promising analytical tool to identify the presence of selected red lake pigments combined with a range of binding media.
\end{abstract}

(C) 2007 Elsevier B.V. All rights reserved.

Keywords: Red lake pigments; Microfluorescence spectroscopy; In situ dye analysis

\section{Introduction}

Anthraquinones and their hydroxy derivatives have been used as red dyes since pre-historic times [1] (Fig. 1), and there are written accounts of their use in ancient Egypt [2]. These red chromophores were extracted from the roots of a variety of plant from the Rubiaceae family (madder family) [1a,3], being alizarin and purpurin the major occurring species in Rubia tinctoria L. (Fig. 1). Purpurin and alizarin have been classified among the

\footnotetext{
* Corresponding author at: Department of Conservation and Restoration, New University Lisbon, Campus da Caparica, Portugal. Tel.: +351 21294 83 22; fax: +351212948322.

** Corresponding author. Tel.: +31 20 3054710; fax: +31 203054700 .

E-mail addresses: mjm@dq.fct.unl.pt (M.J. Melo), Klaas.Jan.vd.Berg@icn.nl (K.J. van den Berg).
}

organic dyes most resistant to light-induced fading [4]. Recent identification of these dyes in more than 1000-years-old archaeological textiles has confirmed this [3]. The red extracts were also prepared to use as a pigment for painting, by precipitating the extract in solution with aluminum salts, such as alum. The pigments were known as "lakes". Madder lake, a mixture of alizarin and purpurin lakes, was widely used in manuscript illuminations [5] and paintings, and is still in use as an artists' material. More recently, these molecules (as chromophores) have found new and high-tech applications, namely in solar energy conversion and development of new imaging materials [6].

During the last decades of the 20th century, there has been a comprehensive body of published work on the physicalchemistry and chemical properties of hydroxy, amino and acylamino derivatives, including significant contributions to the field by Salem and Zewail [7], Gillispie and Marasinghe [8] 

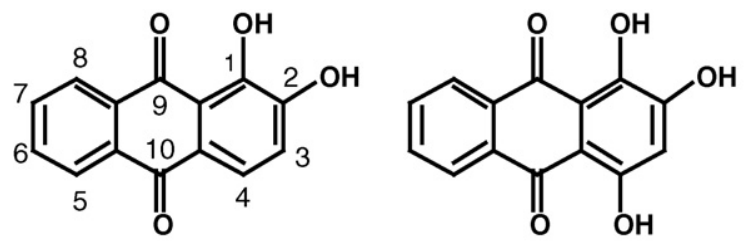<smiles>O=C1C(O)=CC=C2C(=O)c3ccccc3C(O)=C12</smiles>

Fig. 1. Alizarin (1,2-dihydroxy anthraquinone), purpurin (1,2,4-tri-hydroxy anthraquinone) and the 1,10 keto tautomer of alizarin, T. Both, alizarin and purpurin are 9,10 quinones.

and Smith and Barbara [9]. The acidochromic effects in the excited state properties, for purpurin and alizarin, have been recently studied by Favaro and Milliani [10]. From these studies it has been concluded that the substitution pattern of hydroxy anthraquinones determines their excited state properties, where efficient radiationless processes, such as excited state proton transfer (ESPT) and excited state intra- and intermolecular proton transfer (ESIPT) play a determinant role in the overall stability of the molecules; it was shown that the excited-state surface can be sensitively controlled by substituents, allowing the formation of a 1,10 keto tautomer, $\mathrm{T}^{*}$, which ratio (compared to the excited 9,10) can be varied at least two orders of magnitude (Fig. 1); the ESIPT process can be extremely rapid $(<300 \mathrm{fs})$ and was believed to involve proton tunneling [9b]. ESPT and ESIPT with excited state tautomer formation can be considered to induce a photoprotective mechanism for the molecule, enabling a very fast and effective dissipation of the excess energy of the excited state. Light is absorbed; an excited state tautomer is formed (involving an adiabatic proton transfer), and will decay with recovery of the initial ground state molecule. Such photoprotection mechanisms, where energy is absorbed and released by radiationless processes involving $\mathrm{H}$ bond breaking and formation in the excited state, are widely used for UV screeners.

Moreover, the hydroxy derivatives are multi acid-base equilibria systems, interconvertible by $\mathrm{pH}$ inputs (Scheme 1) [10]. Therefore, in the ground state the system is easily characterized through the acid-base equilibrium constants, $\mathrm{p} K_{\mathrm{a}}$, but in the excited state the acid-base equilibria are found to compete with intra- and intermolecular proton transfer (ESIPT) [8-10].

The formation of an excited 1,10 keto tautomer, T*, (Fig. 1) was observed in $\alpha$-hydroxyanthraquinones which lack the 1,4substitution pattern, and the dyes are all weak emitters $[8,9]$. On the other hand, in the presence of a 1,4-substitution pattern, the fluorescence quantum yield was found to be moderate to high<smiles>O=C1c2ccccc2C(=O)c2c1ccc1c2C(=O)[Al]2Oc3c(O)ccc4c3C(=O)O[C@]4(O1)O2</smiles>

Fig. 2. Molecular structure for an $\mathrm{Al}^{3+}$ :alizarin 1:2 complex.

[8a]. The above results are consistent with purpurin acting as a moderate emitter (no ESIPT) and alizarin a very weak one (EISPT present) [8-10]. The fact that ESIPT is suppressed in the case of the 1,4-hydroxy substitution is an intriguing result, and a possible explanation was already proposed by Gillispie in 1984, based on the existence of six-membered pseudo-ring created by the intramolecular hydrogen bonds: "In the 1,4substituted compounds there are two such rings which can interact conjugatively through their common outer ring of the anthraquinone skeleton. Probably this leads to a stabilization of the 9,10-keto form in the $\mathrm{S}_{1}$ (first excited singlet state) sufficiently strong to make ESPT energetically unfavourable" [8a].

While there is significant data from studies of anthraquinone derivatives in solution, less is known about the excited state properties of red lakes (Fig. 2) in the solid state [11]. This is important as these complexes are used for textiles, manuscript illuminations, paintings and other objects that make up cultural heritage. Identification of these materials in art objects, and characterization of their deterioration are of great importance to historians and conservators. However, the fugacity of these materials and the difficulty in obtaining samples for<smiles>O=C1c2ccccc2C(=O)c2c1ccc(O)c2O</smiles> 
analysis may explain why they have received relatively little attention in the literature. Other important red lake pigments are eosin and other fluorescent lakes, that were synthesized in the 19th century, and were used as a pigment for paintings, for example by post-impressionist painter Vincent van Gogh. Eosin lakes are particularly unstable and fade on exposure to light sometimes within a few years (sometimes during the artists' lifetime) [12]. Changes in pigments, whether used pure or mixed with other pigments can alter the appearance of a painting significantly. The identification and state of degradation of lake pigments, in paintings is of fundamental interest since it provides critical information about the artists' aesthetic perspective, conceptions and choices, and how the work has changed with time. Therefore, it is desirable to develop methods that can characterize these materials non-invasively or from small samples that are infrequently available from works of art.

In this paper, the potential of confocal microfluorescence spectroscopy for the characterization of selected red lake pigments and paints based on alizarin, purpurin and eosin is examined. Analytical techniques based on fluorescence detection were selected to take advantage of the opportunity for high sensitivity and selectivity, combined with good spatial resolution [13]. Using fluorescence microscopy in confocal configuration an excitation area of $0.2-0.3 \mu \mathrm{m}$ can be defined for visible radiation with the advantage of similar resolution in three-dimensions [13a]. Fluorescent molecules can also be used to probe and characterize their environment, which is one of the reasons for their popularity in biochemical and pharmaceutical applications and in medicine, for monitoring of species of clinical relevance. Although analytical techniques based on fluorescence detection are widely used, this paper is the first to investigate the potential for emission fluorescence microspectroscopy for the study of red lakes, with the possibility of acquisition of emission and excitation spectra. In the present study, spectra obtained with microfluorescence emission are compared with those acquired using fiber-optic fluorimetry, a set-up currently used in a variety of applications namely, in transportable spectrofluorimeters. Reconstructions of artists' paints made with red lake pigments bound in linseed oil, based on 19th century recipes were examined. Additional test paints were made using the same lake pigments bound in a range of binding media including gum arabic, collagen, PVAc (a vinyl emulsion). Samples were prepared on different supports, including paper and melinex, glass slides and sapphire cells.

A recently commercialized microSPEX spectrofluoremeter (Spex ${ }^{\circledR}$ FluoroMap) was used, that offers the possibility to acquire complete steady-state fluorescence spectra in situ, from samples placed directly on a programmable $x-y-z$ microscope stage. Fluorescence from the sample is directed back up into the microscope. It is also possible to view the fluorescence directly, through a binocular eyepiece and a digital camera. Beam splitting, that enables the separation of emission and excitation light, is achieved using dichroic filters [14]. The optimal conditions for the acquisition of the steady-state spectra will be discussed.

\section{Experimental}

\subsection{Materials}

For the historic reconstructions distilled water, reagent grade chemicals, Madder ground root from Kremer and Eosin, Meister Lucius and Brüning, ICN-reference collection, inv. no. 4328 were used. Linseed oil was obtained from organic linseeds, and prepared as described elsewhere [12].

\subsection{Preparation of the samples}

The set of samples prepared to test the technique can be divided in two categories, model samples and historic reconstructions; in the first category, purpurin and alizarin lakes were bound in each of the following media: gum arabic, parchment glue (collagen) and PVAc emulsion. Lakes made using known ratios of alizarin and purpurin were also set; this is important, because in madder lakes, obtained from natural sources, the ratio between purpurin and alizarin is dependent on the species and methods of preparation. Historic reconstructions were made following 19th century English and French recipes [15], for the red lake pigments, which were then made into linseed oil paints, similar to paints that were found in paintings by Van Gogh [12]. A complete description of the procedures used can be found in the "Supplementary information".

\subsection{Absorption spectroscopy and fluorescence emission measurements}

UV-vis absorption spectra were recorded on a Shimadzu UV2501PC or Cary 100 Bio spectrophotmeter and fluorescence spectra were acquired using a Jovin-Yvon Spex Fluorog 3-2.2 spectrofluorometer. For the measurements with the optic-fiber a SPEX Model F-3000 fiber-optic adaptor was used, and the signal optimized as recommended by the manufacturer. Measurements with the microSPEX (Spex ${ }^{\circledR}$ FluoroMap with Manual Microscope Stage) were obtained with a set-up described elsewhere [14], where the Spex Fluorog 3-2.2 is hyphened to a Olympus BX51M confocal microscope, with spatial resolution controlled with multiple-pinhole turret (pinholes 1-10 corresponding to $2-60 \mu \mathrm{m}$ spot with a $50 \times$ objective). For steady-state fluorescence spectra, a continuous $450 \mathrm{~W}$ xenon lamp, providing an intense, broad spectrum from the UV to near-IR, is directed into a double-grating monochromator. Monochromatic light from the monochromator is fed across a spectrofluorometer-microscope interface into a multiple-pinhole turret of the Olympus BX51 confocal microscope. The incident excitation beam is directed onto the sample, resting on a programmable $x-y-z$ microscope stage. Fluorescence from the sample is directed back up into the microscope. To view the sample's fluorescence directly, a binocular eyepiece and a digital camera are available. Beam-splitting is obtained with dichroic filters; standard dichroic filters, 1-in. diameter $(25 \mathrm{~mm})$, of $478 \mathrm{~nm}, 510,500$ (Glen Spectra) and $570 \mathrm{~nm}$ were used at $45^{\circ}$; they reflect the short wavelengths (excitation) and transmit the long wavelengths (emission). The 
optimization of the signal, through mirror alignment in the optic pathway of the microscope, was performed daily, for all the pinhole apertures, following the manufacturer instructions.

Fluorescence spectra, in homogeneous media, were corrected for the wavelength response of the system; for all the solid state measurements uncorrected spectra are presented. The absorption and emission measurements, in homogeneous media, were performed with $5 \times 10^{-5} \mathrm{M}$ for alizarin/purpurin and with $2 \times 10^{-6} \mathrm{M}$ solutions for the respective lakes. The fluorescence quantum yield for the lakes were determined using purpurin as standard $\left(\phi_{\mathrm{f}}=0.004\right.$ in $\mathrm{MeOH}: \mathrm{H}_{2} \mathrm{O}(75: 25, \mathrm{v}: \mathrm{v})$; the fluorescence quantum yield of purpurin was obtained by comparison with a fluorescein standard solution $\left(\phi_{\mathrm{f}}=0.79\right.$ in $\left.0.1 \mathrm{M} \mathrm{NaOH}\right)$ [16]. The $\phi_{\mathrm{f}}$ value for the purpurin compares well with previously published values, using quinine sulphate as standard [10].

In heterogenous media, for the microSPEX, spectra were acquired after focusing, and for the optic fiber by gentle contact through a black o-ring.

Spectroscopic or equivalent grade solvents and Millipore filtered water were used for all the spectroscopic studies.

The $\mathrm{pH}$ of the solutions was measured with a Metrohm 713 $\mathrm{pH}$ meter or a MetLab pHM240. Calibration was performed with a set of Metrohm buffers of $\mathrm{pH} 4.00$ and 7.00. Adjustment of the desired $\mathrm{pH}$ values was accomplished by addition of $\mathrm{HClO}_{4}$ or $\mathrm{NaOH}$.

Moreover, when studying the fluorescence emission of purpurin and alizarin, the greatest care had to be taken to avoid contamination by metal ions that are able to form fluorescent complexes with the anthraquinone hydroxy derivatives. Low concentration of metal ions, such as $10^{-6} \mathrm{M}$ may induce a significant contribution in the global fluorescence emission in solution where purpurin concentration is typically $\approx 10^{-6}$ to $10^{-7} \mathrm{M}$. Hence, it is advisable to not use toilet paper or analogous for the cleaning of the $\mathrm{pH}$ electrode or other glassware as it contains a large amount of $\mathrm{Al}^{3+}$ ions. In alternative, use filter paper. Also, when studying alizarin lake care should be taken to avoid contamination with purpurin lake, as the excitation and emission spectra are quite similar, since the purpurin lake emission quantum yield is 40 times that of alizarin lake (see below).

\section{Results and discussion}

The results obtained with the historic reconstructions, the paint samples made to resemble 19th century paints, and other model samples will be presented and discussed.

\subsection{Aqueous, homogeneous media}

As there are no comparable published results from studies of the photophysics of the aluminum complexes in solution, a brief study was carried out for purpurin and alizarin in the presence of aluminum ion, $\mathrm{Al}^{3+}$. The alizarin lake is an aluminum two-ligand complex, in which the $\mathrm{Al}$ atom co-ordinates two dye
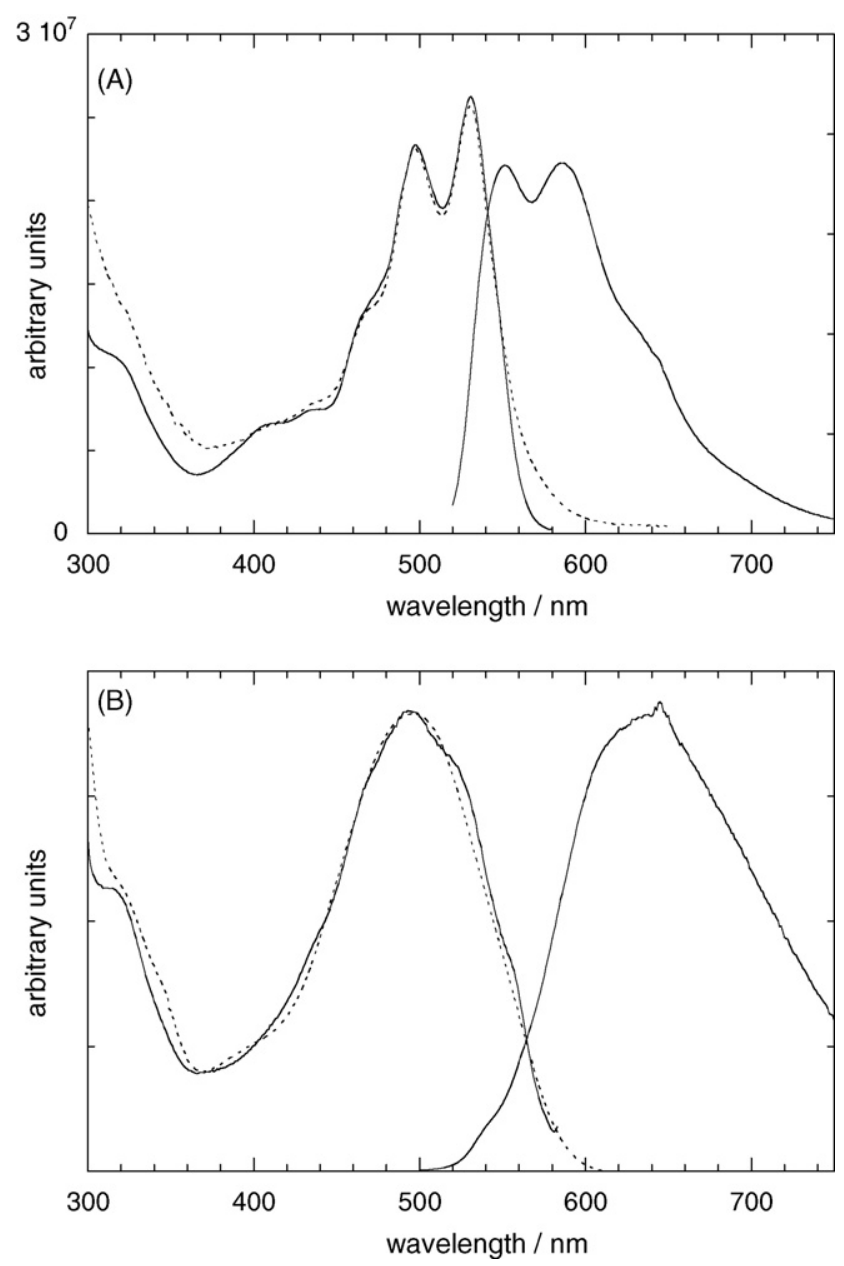

Fig. 3. Corrected emission and excitation spectra $\left(\lambda_{\mathrm{em}}=590 \mathrm{~nm}\right.$ and $\lambda_{\text {exc }}=503 \mathrm{~nm}$; abs at the $\lambda_{\text {exc }}$ for both solutions $\approx 0.018$ ), full line, for purpurin (A) and alizarin (B) aluminum complex, in the presence of a 1:100 excess of $\mathrm{Al}^{3+}$, in a methanol/water solution (75:25\%) at $\mathrm{pH}$ 3.4. Dotted line, absorption spectra.

molecules through the 1-hydroxy oxygen and the adjacent carbonyl oxygen (Fig. 2), forming a six-membered chelate ring structure [17]. Excitation and emission spectra were obtained to be used as reference guides to the understanding of the solid-state behaviour of the paint models (Fig. 3). Fluorescence quantum yields, for the $\mathrm{Al}^{3+}$ chelates, were also obtained and are reported in Table 1, where they can be compared to fluorescence quantum yields of the neutral forms of the respective free chromophores in solution. As expected for fluorogenic ligands where the metal chelates are much more fluorescent than the free ligands themselves [18], the aluminum complexation enables more than a 10 -fold increase in the $\phi_{\mathrm{f}}$ values; the $\phi_{\mathrm{f}}$ of the alizarin aluminum complex compares with the emission quantum yield of neutral form of purpurin, and the purpurin lake displays the strongest emission, and may be considered a moderate to strong emitter. Moreover, the spectra represented in Fig. 3A show a reasonably good mirror-image relationship between the excitation and emission spectra for the purpurin complex, with small Stokes' shift, which can reflect the absence of ESIPT processes. 
Table 1

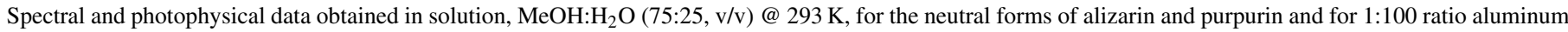
complexes ( $\mathrm{pH} 3.7$ )

\begin{tabular}{|c|c|c|c|}
\hline Alizarin & Purpurin & Alizarin $+\mathrm{Al}^{3+}$ & Purpurin $+\mathrm{Al}^{3+}$ \\
\hline$\lambda_{\max }^{\mathrm{abs}}=430 \mathrm{~nm}(530 ; 570)^{\mathrm{a}}$ & $\lambda_{\max }^{\mathrm{abs}}=480 \mathrm{~nm}(510 ; 545)^{\mathrm{a}}$ & $\lambda_{\max }^{\mathrm{abs}}=500 \mathrm{~nm}$ & $\lambda_{\max }^{\mathrm{abs}}=531 \mathrm{~nm}$ \\
\hline $\begin{array}{l}\varepsilon_{\max }=5830 \mathrm{M}^{-1} \mathrm{~cm}^{-1} \\
\lambda_{\max }^{\text {fluo }}=578 \mathrm{~nm} \\
\phi_{\mathrm{f}}=5.1 \times 10^{-4 \mathrm{~b}}\end{array}$ & $\begin{array}{l}\varepsilon_{\max }=7770 \mathrm{M}^{-1} \mathrm{~cm}^{-1} \\
\lambda_{\max }^{\text {fluo }}=582 \mathrm{~nm} \\
\phi_{\mathrm{f}}=0.004\end{array}$ & $\begin{array}{l}\varepsilon_{\max }=7440 \mathrm{M}^{-1} \mathrm{~cm}^{-1} \\
\lambda_{\max }^{\text {fluo }}=640 \mathrm{~nm} \\
\phi_{\mathrm{f}}=0.006\end{array}$ & $\begin{array}{l}\varepsilon_{\max }=9540 \mathrm{M}^{-1} \mathrm{~cm}^{-1} \\
\lambda_{\max }^{\text {fluo }}=595 \mathrm{~nm} \\
\phi_{\mathrm{f}}=0.29\end{array}$ \\
\hline
\end{tabular}

a Absorption maxima for the $\mathrm{A}^{-}$and $\mathrm{A}^{2-}$ forms of the chromophore.

b Value obtained from Ref. [10].

\subsection{Solid, heterogeneous media}

\subsubsection{Model samples}

The purpurin and alizarin lake model samples were used to test the reproducibility of the microfluorescence data; the model samples used were the $1 \mathrm{~cm}$ square paintings of purpurin and alizarin lakes and their mixtures, in a PVAc emulsion, applied in a Whatmann filter paper, with four replicates for each composition; for each of the prepared paintings 6-9 emission and excitation spectra were acquired, in different days, in different points, and the data was shown to be completely reproducible. Other supports, including melinex sheets, glass slides and sapphire cells, were also used and do not influence the shape of the emission/excitation spectra obtained. To test the potential of the technique as semi-quantitative, mixtures of purpurin and alizarin lakes of known concentration were prepared and the respective spectra acquired (Fig. 4); each concentration point results from a mean value of 36 spectra, obtained in four different experiments $(9 \times 4)$. Taking into account the intrinsic heterogeneity of the solid media, the correlation coefficient of the curve representing the concentration of purpurin in the mixture as a function of the emission intensity can be considered very good, $R^{2}=0.96$ (inset Fig. 4). Also, the emission spectra for the solid state are satisfactory resolved, comparing well with the spectra acquired in solution (Fig. 3A). From Fig. 4 it can be observed that the spectra

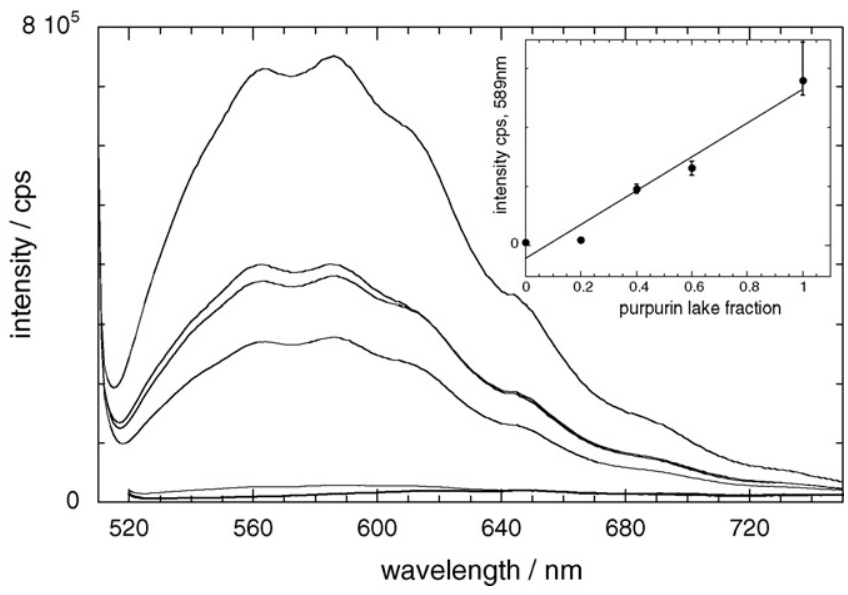

Fig. 4. Micro-emission spectra (not corrected) obtained for mixtures of purpurin and alizarin lakes in a PVA medium, applied in Whatmann filter paper; mixtures, weight:weight, of purpurin:alizarin, 0:100, 20:80, 40:60, 60:40 and 100:0 with $\lambda_{\text {exc }}=490 \mathrm{~nm}$ and dichroic filter of $500 \mathrm{~nm}$. In the inset, the resulting calibration curve with $R^{2}=0.98$ is shown $(y=8212.5 x-61557)$; the calculated uncertainties correspond to a confidence level of $95 \%$. decrease in intensity with the increase of alizarin proportion in the mixture. This is an expected behaviour due to the relative fluorescence yields of the two compounds (see Table 1). Even if it is difficult to see the emission spectra of the alizarin lake with the scale axes of Fig. 4, there is also a blue-shift of the overall spectra since the maximum emission of alizarin is also blue-shifted relative to purpurin. Due to this shift and also to the difference between the fluorescence quantum yields of the two lakes, it was possible to collect the purpurin lake emission directly from the raw data, without any further spectral deconvolution.

Also, the emission spectra acquired for both lakes using parchment glue and arabic gum as binding media compares well to what obtained using PVAc as the binder.

The emission from the model samples was also collected with a fiber-optic adaptor (without microscope) and the results obtained, for four different experiments, are represented in Fig. 5. In this case, for each sample, only one emission spectrum was acquired as the area covered by the fiber-optic, $0.5 \mathrm{~cm}$, takes into account the heterogeneity of the sample. Again, a similar trend in the emission spectra was observed. The linear fitting for the concentration of purpurin in the mixture as a function of the emission intensity was very good, $R^{2}=0.98$ (inset Fig. 5), enabling a semi-quantitative analysis to be carried out.

However, the spectra envelope was less resolved than that obtained with the microSPEX. This could be explained by the

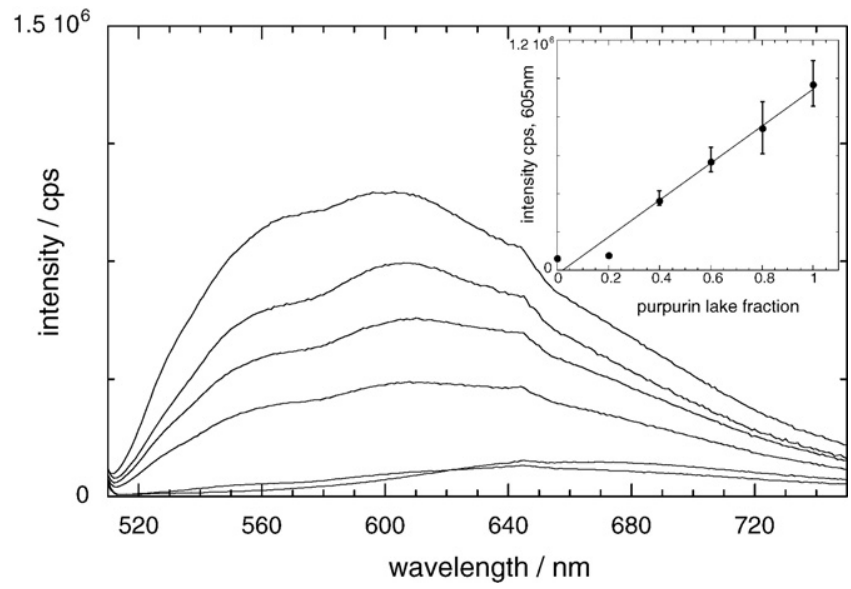

Fig. 5. Emission spectra (not corrected) obtained for mixtures of purpurin and alizarin lakes in a PVA medium, applied in Whatmann filter paper, through fiberoptic; mixtures, weight:weight, of purpurin:alizarin, 0:100, 20:80, 40:60, 60:40, 80:20 and 100:0 with $\lambda_{\text {exc }}=490 \mathrm{~nm}$ and dichroic filter of $500 \mathrm{~nm}$. In the inset, the resulting calibration curve with $R^{2}=0.98$ is shown $(y=9634 x-19537)$; the calculated uncertainties correspond to a confidence level of $95 \%$. 
relatively higher light intensity output of the fiber-optic compared with the microscope, and also that, the light reflected from the sample is more efficiently collected. Therefore, it is possible that interference due to reflection affected the emission spectra obtained with fiber-optic system.

Another disadvantage of using fiber-optic is that it is not possible to acquire excitation spectra of the samples, as it will be the case with the microSPEX; this will be described in the forthcoming discussion.

In the insets of Figs. 4 and 5, the results and associated uncertainties are given considering a confidence level of $95 \%$. The detection limits found are $\approx 11 \%$ for both cases, MicroSPEX and fiber-optic (calculated as three times the standard deviation of the sample where the amount of purpurin was 0\%-blank signal [19]). This reveals that below this percentage of purpurin in the alizarin:purpurin mixture there is a significant degree of uncertainty in the measurements.

\subsubsection{Historical reconstructions}

3.2.2.1. Kopp's $A l^{3+}$ lakes. Fig. 6 shows the emission and excitation spectra from the historic reconstructions for Kopp's Purpurin lake, where lead white was applied as an underlayer and the lake is applied in oil media. The emission spectra were obtained using 510 and $570 \mathrm{~nm}$ dichroic filters, and it was concluded that the $570 \mathrm{~nm}$ cannot be used for this red lakes as part of the emission is cut-off, with consequent loss of information that can induce to a misinterpretation of the experimental results. On the other hand, it was possible to obtain good excitation spectra with the $570 \mathrm{~nm}$ dichroic filter. The excitation spectra reproduce the absorption spectra of the sample, enabling a better identification of the chromophore. This is an important advantage in the field of cultural heritage, since in ancient samples, complex aged mixtures can display also a complex emission spectra, and having access to a well resolved excitation spectra will facilitate the characterization of the chromophores present.

All the spectra represented in Fig. 6 were obtained with high spatial resolution ( $8 \mu \mathrm{m}$ spot) using pinhole 5 . The same conditions were used to collect the emission for the same sample in

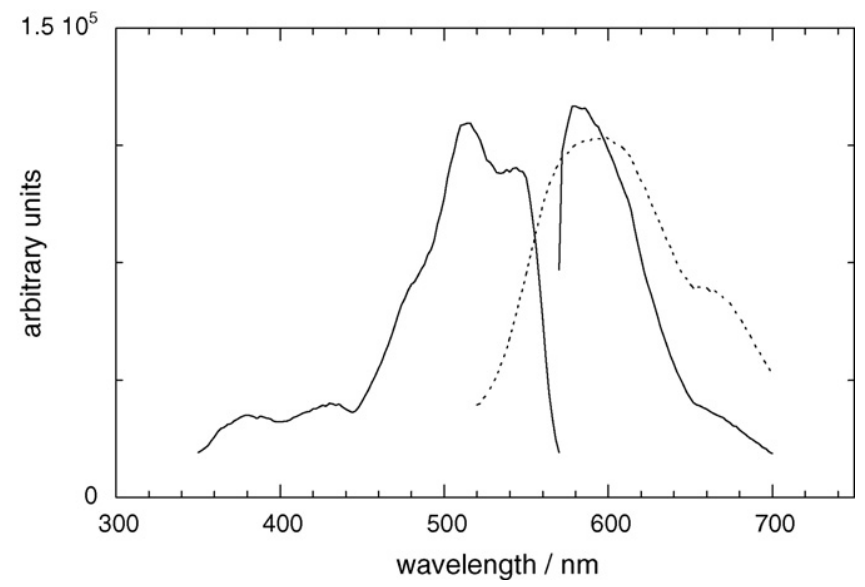

Fig. 6. Kopp's purpurin lake emission ( 570 - full line - and $510 \mathrm{~nm}$ - dotted line - dichroics) and excitation ( $570 \mathrm{~nm}$ dichroic) spectra $(8 \mu \mathrm{m}$ spot $)$.

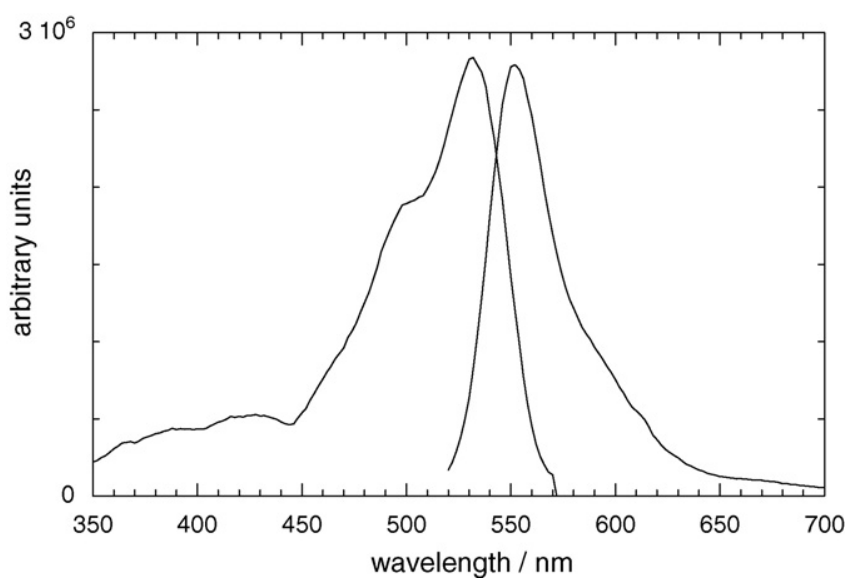

Fig. 7. Eosin lake emission and excitation $(570 \mathrm{~nm}$ dichroic) spectra with an $8 \mu \mathrm{m}$ spot.

a resin, as a model for cross sections of painting samples. The results acquired were analogous to those presented in Fig. 5.

3.2.2.2. Eosin $A l^{3+}$ lakes. The emission and excitation spectra for the eosin lakes were obtained, as for Kopp's Purpurin lake, from a cross section, in $8 \mu \mathrm{m}$ and $4 \mu \mathrm{m}$ spots, and representative emission and excitation spectra are given in Fig. 7. Here, again, it was possible to acquire an excitation spectrum with the $570 \mathrm{~nm}$ dichroic filter. Besides, as expected, being eosin a strong fluorophore, the emission intensity is circa one order of magnitude higher than that obtained for Kopp's lake.

\subsubsection{3. $G E O^{\odot}$ fluorescent colors. The spatial resolution of the} technique was tested in a complete cross-section, were a paint sample with a chalk $\left(\mathrm{CaCO}_{3}\right)$ preparation and a white lead $\left(\mathrm{PbCO}_{3} \cdot \mathrm{Pb}(\mathrm{OH})_{2}\right)$ imprimatura was used for a fluorescent vinyl commercial paint, $\mathrm{GEO}^{\odot}$, inset Fig. 8. Again remarkably good excitation and emission spectra were acquired with a high spatial resolution of $8 \mu \mathrm{m}$.

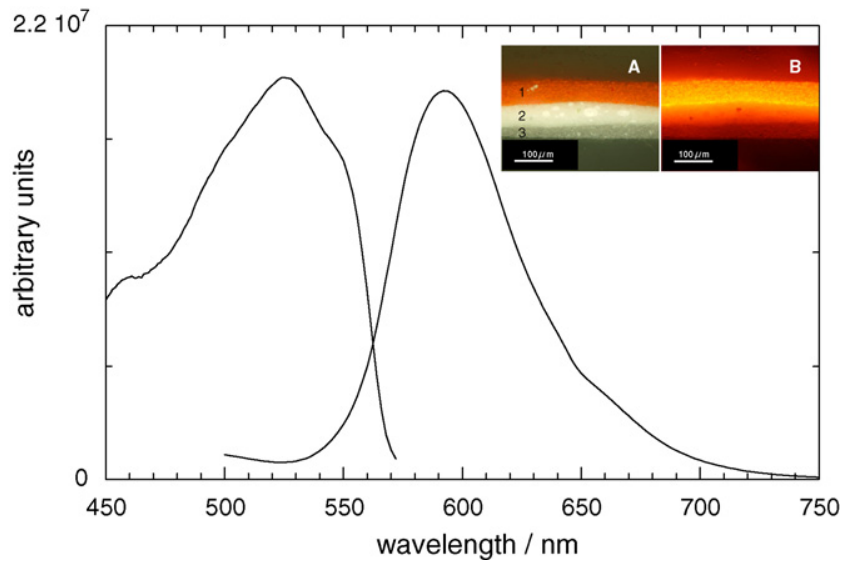

Fig. 8. In the inset, GEO fluorescent vinyl paint (1) over a chalk preparation (3) and white lead imprimatura (2), in a ester resin cross section: (A) image acquired with visible light and (B) exciting with green light (filter set 14-BP510-560 FT580 LP590). The emission and excitation spectra were obtained under excitation with an $8 \mu \mathrm{m}$ spot, using the $570 \mathrm{~nm}$ dichroic filter. 


\section{Conclusions}

Micro-emission molecular fluorescence proved to be a promising analytical tool to identify the presence of selected red lake pigments combined with a range of binding media. Red lakes that displayed characteristic emission spectra included purpurin and eosin-based pigments although the potential for identification of lake pigments other than those examined in the present study may apply to a wider range of artists' materials. The radiation used is of low intensity and non-destructive to the molecules. Analysis may be carried out in situ, without sampling, or on prepared paint cross-sections that offers the opportunity to look at materials in discrete layers of a painting. The acquisition of spectra is rapid, with high spatial resolution and the data reliable and reproducible. Together with full emission spectra, it was possible to acquire well-resolved excitation spectra, which is a great advantage when studying complex aged samples. The present investigation suggests that micro-emission fluorescence can also be used as a semi-quantitative method for madder lakes, enabling the determination of purpurin lake fraction in a mixture of purpurin and alizarin.

The analytical method developed here for the identification of red lakes prepared as aluminum complexes with purpurin and eosin defines the optimal conditions for the acquisition of the steady-state emission fluorescence spectra using a $500 \mathrm{~nm}$ or $510 \mathrm{~nm}$ dichroic filter, with excitation at $490 \mathrm{~nm}$ for the red anthraquinone lakes. For eosin type lakes the $570 \mathrm{~nm}$ dichroic filter was used, and excitation was performed at the absorption maxima. All steady-state excitation spectra were acquired with the $570 \mathrm{~nm}$ dichroic. The experimental set-up employed a pinhole $5(8 \mu \mathrm{m})$ to define the area analyzed in cross sections. This spatial resolution is appropriate for the analysis of individual pigments particles or aggregates in a paint film. Generally, it offers an optimized signal to noise ratio and good spatial resolution for artists' paints. For the eosin type compounds it was possible to acquire spectra with smaller pinholes apertures $(4 \mu \mathrm{m})$. When spatial resolution was not an important requirement a pinhole 8 $(30 \mu \mathrm{m})$ was selected. Another method to be explored might be to gather data from selected areas, thus providing the opportunity to characterize materials in paint that contains mixtures of pigments in different layers.

Although the molecular structures of lakes are difficult to determine by fluorescence emission alone, combined with data from other analytical techniques, information about the environment [20] of the colorant and its state of degradation can be obtained. Work is currently in progress on the analysis of red lakes in cross sections, aiming to extend the application of the technique for a wider range of lake pigments in different states of degradation, in samples from painted works of art. Exploring the in-depth profiling, future work will investigate the application of this non-destructive technique for analysis of red lakes on actual works of art, even when varnished.

With regard to the comparison of the method with the currently available transportable fiber-optic spectrofluorimeters, the more significant advantages of micro-emission molecular fluorescence are: (i) the possibility to acquire both emission and excitation spectra, (ii) high spatial resolution and also (iii) in- depth profiling; this last feature was not explored in this work. Moreover, this work shows that the quality of the spectral information, namely the spectral resolution and the signal to noise ratio, is very high when compared to those obtained with the fiber-optic spectrofluorimeters, as was shown in this work. It is important to stress the fact that, an excitation spectrum reproduces the chromophore absorption spectrum, a relevant parameter for the characterization of a dye or a pigment. The simultaneous acquisition of emission and excitation (absorption) spectra together with the high spectral resolution will facilitate a more accurate identification of dye molecules and lakes.

\section{Acknowledgments}

This work was supported by the Portuguese Science Foundation and FEDER, through the Associate Laboratory for Green Chemistry and the projects POCI/QUI/55672/2004 "The Molecules of Color in Art: a Photochemical Study" and POCTI/EAT/33782/2000 "An interdisciplinary approach to the study of color in Portuguese manuscript illuminations".

\section{Appendix A. Supplementary data“Supplementary}

Supplementary data associated with this article can be found, in the online version, at doi:10.1016/j.talanta.2007.07.036.

\section{References}

[1] (a) D. Cardon, Le monde des Teintures naturelles, 2nd ed., Belin, Paris, 2003;

(b) J. Wouters, N. Rosario-Chirinos, JAIC 31 (1992) 237.

[2] Halleux, R. (Ed.), Les Alchimistes Grecs: Papyrus de Leyde (1st ed., 1981), Papyrus de Stockholm, Recettes, Les Belles Lettres, Paris, 2002.

[3] (a) D. Cardon, Teintures précieuses de la Méditerranée: Poupre $\sim$ Kermès $\sim$ Pastel, Musée des Beaux-Arts de Carcassonne, Centre de Documentació I Museu Tèxtil de Terrassa, Carcassonne, 1999;

(b) E.W. Fitzhugh (Ed.), Artists' Pigments, A Handbook of their History and Characteristics, vol. 3, National Gallery of Art, Washington, 1997;

(c) B. Szostek, J. Orska-Gawrys, I. Surowiec, M. Trojanowicz, J. Chromatogr. A 1012 (2003) 179;

(d) X. Zhang, R.A. Laursen, Anal. Chem. 77 (2005) 2022.

[4] D.K. Palit, H. Pal, T. Mukherjee, J.P. Mittal, J. Chem. Soc. Faraday Trans. 86 (1990) 3861.

[5] (a) C. Cennini, Il Libro dell' Arte, In: F. Frezzato (Ed.), Neri Pozza Editore, Vicenza, 2003;

(b) F. Brunello (Ed.), De Arte Illuminandi e altri trattati sulla miniatura medievale, Neri Pozza Editore, Vicenza, 1992;

(c) S. Blondheim, JQR 19 (1928) 97.

[6] (a) R. Huber, J. Moser, M. Gratzel, J. Wachtweitl, J. Phys. Chem. B 106 (2002) 6494;

(b) J. Kim, J. Kang, D. Han, C. Lee, K. Ahn, Chem. Mater. 10 (1998) 2332.

[7] M.S. El Ezaby, T.M. Salem, A.H. Zewail, R. Issa, J. Chem. Soc. B 7 (1970) 1293.

[8] (a) M.H. Van Benthem, G.D. Gillispie, J. Phys. Chem. 88 (1984) 2954; (b) P.A.B. Marasinghe, G.D. Gillispie, Chem. Phys. 136 (1989) 249.

[9] (a) T.P. Smith, K.A. Zaklika, K. Thakur, G.C. Walker, K. Tominaga, P.F. Barbara, J. Phys. Chem. 95 (1991) 10465;

(b) R.A. Denny, B. Bagchi, P.F. Barbara, J. Chem. Phys. 115 (2001) 6058.

[10] C. Miliani, A. Romani, G. Favaro, J. Phys. Org. Chem. 13 (2000) 141. 
[11] (a) E.R. de la Rie, Stud. Conserv. 27 (1982) 1;

(b) A. Favaro, C. Miliani, G. Romani, M.J. Vagnini, Chem. Perkin Soc. Trans. 2 (13) (2002) 192;

(c) T. Del Giacco, L. Latterini, F. Elisei, Photochem. Photobiol. Sci. 2 (2003) 681-687.

[12] A. Burnstock, I. Lanfear, K.J. van den Berg, L. Carlyle, M. Clarke, E. Hendriks, J. Kirby, Preprints of the 14th Triennial Meeting of the ICOM Committee for Conservation, vol. 1, The Hague, 2005, p. 459.

[13] (a) B. Valeur, Molecular Fluorescence: An Introduction—Principles and Applications, Wiley-VCH, Weinheim, 2002;

(b) C. Clementi, C. Miliani, A. Romani, G. Favaro, Spectrochim. Acta A 64 (2006) 906;

(c) E. Destandau, V. Alain, É. Bardez, Anal. Bional. Chem. 378 (2004) 402;

(d) J. Seixas de Melo, A.P. Moura, M.J. Melo, J. Phys. Chem. A. 108 (2004) 6975.

[14] http://www.biosciencetechnology.com/ShowPR.aspx?PUBCODE=090 $\&$ ACCT $=9000003025 \&$ ISSUE $=0307 \&$ RELTYPE $=$ PR \&ProdCode $=$ 00005878\&PRODLETT $=\mathrm{A}$.
[15] J. Kirby, in: M. Clarke, J.H. Townsend, A. Stijnman (Eds.), Postprints of the Conference Approaching the Art of the Past: Sources and Reconstructions, Archetype, London, 2005, pp. 69-77.

[16] R.E. Kellogg, R.G. Bennett, J. Chem. Phys. 41 (1964) 3042.

[17] A.E. Martell, R.M. Smith, R.J. Moteikatis, NIST Critical Stability Constants of Metal Complexes Database NIST Standard Reference Databases, version 6.0, NIST, Gaithersburg, MD, 2001, p. 20899.

[18] E. Bardez, V. Alain, E. Destandau, A. Fedorov, J.M.G. Martinho, J. Phys. Chem. A 105 (2001) 10613.

[19] D.C. Harris, Quantitative Chemical Analysis, 6th ed., W.H. Freeman and Company, New York, 2003.

[20] (a) A.P. de Silva, P. Tecilla, J. Mater. Chem. 15 (2005) 2637;

(b) D.C. Magri, J.F. Callan, A.P. de Silva, D.B. Fox, N.D. McClenaghan, K.R.A.S. Sandanayake, J. Fluorescence 15 (2005) 769;

(c) S. Uchiyama, Y. Matsumura, A.P. de Silva, K. Iwai, Anal. Chem. 76 (2004) 1793;

(d) Y.-L. Wei, J.-Q. Li, C. Dong, S.-M. Shuang, D.-S. Liu, C.W. Huie, Talanta 70 (2006) 37. 